\title{
Simultaneous State and Unknown Input Estimation for Complex Networks with Redundant Channels under Dynamic Event-Triggered Mechanisms
}

\author{
Qi Li, Member, IEEE, Zidong Wang, Fellow, IEEE, Jun Hu, Member, IEEE, and Weiguo Sheng, Member, IEEE
}

\begin{abstract}
This paper addresses the simultaneous state and unknown input estimation problem for a class of discrete timevarying complex networks (CNs) under redundant channels and dynamic event-triggered mechanisms (ETMs). The redundant channels, modeled by an array of mutually independent Bernoulli distributed stochastic variables, are exploited to enhance the transmission reliability. For energy-saving purposes, a dynamic event-triggered transmission scheme is enforced to ensure that every sensor node sends its measurement to the corresponding estimator only when a certain condition holds. The primary objective of the investigation carried out is to construct a recursive estimator for both the state and the unknown input such that certain upper bounds on the estimation error covariances are first guaranteed and then minimized at each time instant in the presence of dynamic event-triggered strategies and redundant channels. By solving two series of recursive difference equations, the desired estimator gains are computed. Finally, an illustrative example is presented to show the usefulness of the developed estimator design method.
\end{abstract}

Index Terms-Complex networks; dynamic event-triggered mechanisms; state and input estimation; redundant channels; recursive algorithm.

\section{INTRODUCTION}

The state estimation (SE) or filtering problem has always been an active research topic because of its wide applications in signal processing and systems science, see e.g. [6], [7], [13], [23], [32], [33]. In order to deal with different types of exogenous disturbances, a variety of SE techniques have been developed with examples including Kalman estimation approach [4], [18], [37], variance-constrained estimation method [16], [17], [23], $H_{\infty}$ estimation scheme [38], [40], [46] and set-membership estimation technique [28], [41], [43], [48]. In these SE strategies, it is typically required that all inputs (e.g. fault or unmodeled dynamics) of the underlying system

This work was supported in part by the National Natural Science Foundation of China under Grants 62003121, 61873082, 61873148 and 61933007, the Zhejiang Provincial Natural Science Foundation of China under Grant LQ20F030014, the Outstanding Youth Science Foundation of Heilongjiang Province of China under grant JC2018001, the Fundamental Research Foundation for Universities of Heilongjiang Province of China under Grant 2019KYYWF-0215, the Royal Society of the UK, and the Alexander von Humboldt Foundation of Germany. (Corresponding author: Weiguo Sheng.)

Q. Li and W. Sheng are with the School of Information Science and Engineering, Hangzhou Normal University, Hangzhou 311121, China. (Email: weiguouk@hotmail.com)

Z. Wang is with the Department of Computer Science, Brunel University London, Uxbridge, Middlesex, UB8 3PH, United Kingdom. (Email: Zidong.Wang@brunel.ac.uk)

$\mathrm{J}$. Hu is with the Department of Mathematics, Harbin University of Science and Technology, Harbin 150080, China. should be known. However, in many practical situations, certain inputs are inevitably unknown owing probably to the expensive/unbearable cost of acquiring the information of the inputs. For instance, in a machine tool system, the cutting force exerted by the tool is generally difficult to measure, which can be regarded as an unknown input estimated based on the available measurements [10]. As such, it is of great importance to propose a new SE scheme that is capable of estimating the unknown input and system state simultaneously. Accordingly, over the past few decades, a large amount of effort has been devoted to the investigation of joint input and SE problem, see [14], [49] for some representative works.

Complex networks (CNs) have recently received a rapidly growing research interest because of its capability of describing various kinds of real-world systems such as social networks, neural networks, World Wide Web and scientific collaboration networks [1], [2], [31]. Typically, a CN consists of large numbers of nodes with highly interconnected relationships, by which every node can be treated as a subsystem whose dynamical behavior is affected by other nodes. In the past years, in order to better understand the internal characteristic of $\mathrm{CNs}$, the $\mathrm{SE}$ problem for CNs has become a primary research focus and a great number of excellent results have been available in the literature, see e.g. [8], [24], [35], [39] and the reference therein. As for the problem of simultaneous state and input estimation, the relevant results have been quite few despite the initial effort made in [44], where the problem of joint SE and unknown input reconstruction has been handled for uncertain time-invariant CNs. As a matter of fact, almost all practical systems possess certain time-varying characteristics and, consequently, there is a practical need to design joint state and unknown input estimation (SUIE) schemes for time-varying CNs.

In many applications, due primarily to the bandwidth restriction and random fluctuation of network channels, those signals transmitted from the system to the sensor node through only one channel would inevitably suffer from the random packet losses, and this might lead to severe degradations of the overall system performance [9], [16]. In the past few years, many researchers have attempted to remedy the undesired effects from the random packet losses [3], [5]. For example, the scheme of redundant transmission channels, which contains two or more available communication accesses, has been developed in [29] whose main advantage lies in the extra guarantee of successful data transmission if certain channel fails to operate, thereby effectively improving the communication reliability. 
Recently, the redundant channel schemes have been widely applied in networked systems [40], [47], sensor networks and multiagent systems [42] to deal with the sliding mode control, the distributed filtering and the $H_{\infty}$ consensus problems, respectively. Regarding CNs, the generalized SE method has been presented in [36], where both the redundant channels and Round-Robin protocol have been taken into consideration.

As an effective means of saving energy, the event-triggered transmission strategy has attracted much research attention from the control community because of its capability of reducing unnecessary transmissions [22], [45]. By employing this transmission mechanism, a signal is transmitted only after the occurrence of a certain pre-defined event. In recent years, considerable progress has been made on the event-triggered SE problems for different types of systems, see e.g. [11], [12], [15], [21], [34]. Particularly, in [15], a dynamic event-triggered mechanism (ETM), which introduces an auxiliary dynamical equation into the traditional ETM, has been proposed to save resource even further without significantly degrading the system performance. In fact, the dynamic event-triggered transmission scheme has recently become more and more popular, and much work has been done along this line, see e.g. [20], [50] for some latest literature. It is noticeable that there have been very few results on the joint SUIE problem for time-varying CNs under dynamic ETMs yet, and this gives rise to the main motivation of the current investigation.

In view of the above discussions, we endeavor to handle the joint SUIE problem for a class of time-varying CNs with redundant channels and dynamic ETMs. In particular, the redundant channels are introduced to increase the communication reliability and the dynamic ETMs are employed to determine when sensors transmit their own measurements to the corresponding estimators. There are three main difficulties for the considered research issue, namely, 1) the establishment of certain criterion for deriving certain upper bounds on the error covariances of input and SE; 2) the design of suitable estimators for both the input and state such that the derived upper bounds can be optimized at each time instant; and 3) the examination of the impacts from unknown input, dynamic ETMs and redundant channels on the estimation performance. The main contributions are summarized as follows: 1) a new joint SUIE scheme is presented for time-varying CNs subject to redundant channels and dynamic ETMs; 2) a recursive induction approach is developed to guarantee the existence of upper bounds on the error covariances of input and SE; and 3) the desired estimator gains are determined in the sense of minimizing the obtained upper bounds (on the estimation error covariances) that are parameterized by means of the solutions to certain recursive difference equations. Furthermore, the primary differences of the results developed for $\mathrm{CNs}$ in this paper from [19] include: 1) the problem of state and input estimation is investigated simultaneously; and 2) the effects of unknown input, dynamic ETMs as well as redundant channels are concurrently considered in the estimator design.

Notations: $\mathbb{R}^{n}$ denotes the $n$-dimensional Euclidean space. $\mathbb{R}^{n \times m}$ stands the set of all $n \times m$ real matrices. $A^{T}$ represents the transposition of the matrix $A$ and $\|\cdot\|$ is the Euclidean norm. $I$ denotes the identity matrix and $\operatorname{diag}\{\cdots\}$ refers to a block-diagonal matrix. $\mathbb{E}\{a\}$ is the expectation of the stochastic variable a. Let $\mathrm{R}(\cdot)$ be the rank of a matrix. $\mathrm{P}\{\cdot\}$ denotes the probabilities of ".". For a real symmetric matrix $\mathrm{P}, \mathrm{P} \succeq 0(\mathrm{P} \succ 0)$ means that the matrix $\mathrm{P}$ is positive semidefinite (positive definite).

\section{Problem Formulation}

Consider a class of discrete time-varying CNs defined on a finite horizon $k \in[0, N]$ :

$$
\begin{aligned}
x_{i, k+1}= & f_{k}\left(x_{i, k}\right)+\sum_{j=1}^{n} \omega_{i j} \Upsilon x_{j, k}+A_{i, k} d_{i, k} \\
& +B_{i, k} w_{k}, \quad(i=1,2, \ldots, n)
\end{aligned}
$$

where $x_{i, k} \in \mathbb{R}^{n_{x}}$ and $d_{i, k} \in \mathbb{R}^{n_{d}}$ are the state vector and the unknown input vector of the $i$ th node, respectively. $W=$ $\left(\omega_{i j}\right)_{n \times n}$ is the outer-coupling matrix whose non-diagonal elements satisfy $\omega_{i j} \geq 0$ but not all zeros and the diagonal elements satisfy $\omega_{i i}=-\sum_{j=1, j \neq i}^{n} \omega_{i j}$ with $W=W^{T}$. $\Upsilon=\operatorname{diag}\left\{\iota_{1}, \iota_{2}, \ldots, \iota_{n_{x}}\right\}$ is an inner-coupling matrix. $A_{i, k}$ and $B_{i, k}$ are known time-varying matrices. $w_{k} \in \mathbb{R}^{n_{w}}$ is the process noise with zero-mean and covariance $R_{k}>0$. The initial value of the unknown input is set as $d_{i, 0}=\bar{d}_{i}$.

Assumption 1: [30] The nonlinear function $f_{k}(\cdot)$ in (1) satisfies $f_{k}(0)=0$ and

$$
\left\|f_{k}(\imath)-f_{k}(\jmath)-E_{k}(\imath-\jmath)\right\| \leq \ell_{k}\|\imath-\jmath\|
$$

for all $\iota, \jmath \in \mathbb{R}^{n_{x}}$, where $\ell_{k}$ is a known nonnegative scalar and $E_{k}$ is a known matrix.

The measurement output with redundant channels is expressed as

$$
\begin{aligned}
y_{i, k}= & \pi_{i, k}^{1} C_{i, k}^{1} x_{i, k}+F_{i, k} d_{i, k}+D_{i, k} v_{k} \\
& +\sum_{p=2}^{z}\left\{\prod_{q=1}^{p-1}\left(1-\pi_{i, k}^{q}\right) \pi_{i, k}^{p} C_{i, k}^{p}\right\} x_{i, k}, \\
& (i=1,2, \ldots, n)
\end{aligned}
$$

where $y_{i, k} \in \mathbb{R}^{n_{y}}$ is the measurement output of the $i$ th node, $C_{i, k}^{p}(p=1,2, \ldots, z), D_{i, k}$ and $F_{i, k}$ are known matrices with $\mathrm{R}\left(F_{i, k}\right)=n_{d}, v_{k} \in \mathbb{R}^{n_{v}}$ is the measurement noise with mean being zero and covariance being $Q_{k}>0$. The stochastic variable $\pi_{i, k}^{p}$, which describes the packet dropout phenomenon in the $p$ th channel, satisfies

$$
\begin{aligned}
& \mathrm{P}\left\{\pi_{i, k}^{p}=0\right\}=1-\bar{\pi}_{i}^{p}, \\
& \mathrm{P}\left\{\pi_{i, k}^{p}=1\right\}=\bar{\pi}_{i}^{p}
\end{aligned}
$$

with $\bar{\pi}_{i}^{p} \in[0,1]$ being a known scalar.

Remark 1: By restoring to the Bernoulli distributed stochastic variables $\pi_{i, k}^{p}(p=1,2, \ldots, z)$, the measurement model (3) for node $i$ is capable of depicting the phenomenon of $z$-channel packet dropouts, where the priority of these $z$ channels is ranked in a descending order from channel 1 to channel $z$. Furthermore, in order to save communication resources and avoid data collisions, it is assumed that, at each time instant, only one (or none) channel among the $z$-channels would be activated to transmit the measurement output. Accordingly, the values of $\pi_{i, k}^{p}$ can fall into the following three 
cases: 1) if $\pi_{i, k}^{1}=1$, then the measurement is delivered via the first channel; 2) for any given integer $s(s=2, \ldots, z)$, if $\pi_{i, k}^{s}=1$ and $\pi_{i, k}^{l}=0$ for all $l=1,2, \ldots, s-1$, then the measurement is delivered via the sth channel and the packet dropouts occur at all previous $s-1$ channels; and 3) if $\pi_{i, k}^{p}=0$ for all $p=1,2, \ldots, z$, then all channels are useless and the packet dropouts for node $i$ occur. Compared to the traditional one-channel case, it is obvious that the redundant channel communication strategy could reduce the occurrence probability of packet dropouts, thereby effectively improving the network reliability. Note that such a measurement model with $z$ redundant channels has been widely used in the existing literature, see e.g. [42].

Assumption 2: $x_{i, 0}$ is a stochastic variable with mean $\bar{x}_{i, 0}$ and covariance $P_{i, 0}^{x}>0$. Furthermore, all the stochastic variables $\pi_{i, k}^{1}, \ldots, \pi_{i, k}^{z}, x_{i, 0}, w_{k}$ and $v_{k}$ in this paper are mutually independent.

For energy-saving purposes, a dynamic ETM is used for each node to decide when to transmit the measurement. Denote by $0 \leq \kappa_{0}^{i}<\kappa_{1}^{i}<\cdots<\kappa_{l}^{i}<\cdots$ the triggering instant sequence with $\kappa_{l+1}^{i}$ determined by

$$
\kappa_{l+1}^{i}=\min \left\{k \mid k>\kappa_{l}^{i}, \frac{1}{\mu_{i}} \xi_{i, k}+\sigma_{i}-\left\|\psi_{i, k}\right\| \leq 0\right\} .
$$

Here, $\sigma_{i}$ and $\mu_{i}$ are given positive scalars, $\psi_{i, k}$ is defined by $\psi_{i, k} \triangleq y_{i, k}-y_{i, \kappa_{l}^{i}}$ with the latest transmitted measurement $y_{i, \kappa_{l}^{i}}$, and $\xi_{i, k}$ is a dynamic variable satisfying

$$
\xi_{i, k+1}=\gamma_{i} \xi_{i, k}+\sigma_{i}-\left\|\psi_{i, k}\right\|, \quad \xi_{i, 0}=\xi_{0}^{i}
$$

where $\xi_{0}^{i} \geq 0$ is the given initial condition and $\gamma_{i}$ is a suitable positive scalar. Letting the parameters $\gamma_{i}$ and $\mu_{i}$ satisfy $\gamma_{i} \mu_{i} \geq$ 1 , the variable $\xi_{i, k}$ satisfies $\xi_{i, k} \geq 0$ for all $k \in[0, N]$.

Remark 2: It is easily seen from (5) and (6) that the dynamic variable $\xi_{i, k}$ could regulate the threshold value of the dynamic triggering condition according to the event error $\left\|\psi_{i, k}\right\|$. Specifically, if $\left\|\psi_{i, k}\right\|$ is increasing, then $\xi_{i, k+1}$ would start decreasing and vise verse, which is reasonable in both theory and practice. Moreover, another introduced parameter $\mu_{i}$ in (5) is a given positive scalar that influences the transmission frequency and the transmission frequency would increase as $\mu_{i}$ increases. Note that the static ETMs considered in [23] can be seen as special cases of the dynamic ETMs utilized in this paper when $\mu_{i}$ tends to infinity.

Remark 3: The idea of dynamic ETM was first proposed in [15] for the control problems, which recently has been adopted in the literature to tackle with various control/filtering issues, see e.g. [20]. Note that this paper represents the first attempt to introduce a dynamic event-triggering strategy into the SUIE problem for CNs. By employing the dynamic ETM (5), the needless data transmissions could be effectively reduced and hence the resource consumption is alleviated.

Based on the dynamic event-triggered measurement, the estimators for node $i(i=1,2, \ldots, n)$ are constructed as follows:

$$
\hat{x}_{i, k+1 \mid k}=f_{k}\left(\hat{x}_{i, k \mid k}\right)+\sum_{j=1}^{n} \omega_{i j} \Upsilon \hat{x}_{j, k \mid k}+A_{i, k} \hat{d}_{i, k},
$$

$$
\begin{aligned}
\hat{x}_{i, k+1 \mid k+1}= & \hat{x}_{i, k+1 \mid k}+K_{i, k+1}\left(y_{i, \kappa_{s}^{i}}-\bar{\pi}_{i}^{1} C_{i, k+1}^{1} \hat{x}_{i, k+1 \mid k}\right. \\
& -\sum_{p=2}^{z}\left\{\prod_{q=1}^{p-1}\left(1-\bar{\pi}_{i}^{q}\right) \bar{\pi}_{i}^{p} C_{i, k+1}^{p}\right\} \hat{x}_{i, k+1 \mid k} \\
& \left.-F_{i, k+1} \hat{d}_{i, k+1}\right), \\
\hat{d}_{i, k+1}= & G_{i, k+1}\left(y_{i, \kappa_{s}^{i}}-\bar{\pi}_{i}^{1} C_{i, k+1}^{1} \hat{x}_{i, k+1 \mid k}\right. \\
& \left.-\sum_{p=2}^{z}\left\{\prod_{q=1}^{p-1}\left(1-\bar{\pi}_{i}^{q}\right) \bar{\pi}_{i}^{p} C_{i, k+1}^{p}\right\} \hat{x}_{i, k+1 \mid k}\right)
\end{aligned}
$$

for $k+1 \in\left[\kappa_{s}^{i}, \kappa_{s+1}^{i}\right)(s \geq 0)$. Here, $\hat{x}_{i, k+1 \mid k}$ is the onestep prediction, $\hat{x}_{i, k \mid k}$ is the estimate of state $x_{i, k}, \hat{d}_{i, k}$ is the estimate of the unknown input $d_{i, k}$ at time $k$, and $K_{i, k+1}$ and $G_{i, k+1}$ are estimator gains to be designed.

By the definition of $\psi_{i, k},(7)$ is transformed into

$$
\begin{aligned}
\hat{x}_{i, k+1 \mid k}= & f_{k}\left(\hat{x}_{i, k \mid k}\right)+\sum_{j=1}^{n} \omega_{i j} \Upsilon \hat{x}_{j, k \mid k}+A_{i, k} \hat{d}_{i, k}, \\
\hat{x}_{i, k+1 \mid k+1}= & \hat{x}_{i, k+1 \mid k}+K_{i, k+1}\left[y_{i, k+1}-\bar{\pi}_{i}^{1} C_{i, k+1}^{1} \hat{x}_{i, k+1 \mid k}\right. \\
& -\sum_{p=2}^{z}\left\{\prod_{q=1}^{p-1}\left(1-\bar{\pi}_{i}^{q}\right) \bar{\pi}_{i}^{p} C_{i, k+1}^{p}\right\} \hat{x}_{i, k+1 \mid k} \\
& \left.-\psi_{i, k+1}-F_{i, k+1} \hat{d}_{i, k+1}\right], \\
\hat{d}_{i, k+1}= & G_{i, k+1}\left[y_{i, k+1}-\psi_{i, k+1}-\bar{\pi}_{i}^{1} C_{i, k+1}^{1} \hat{x}_{i, k+1 \mid k}\right. \\
& \left.-\sum_{p=2}^{z}\left\{\prod_{q=1}^{p-1}\left(1-\bar{\pi}_{i}^{q}\right) \bar{\pi}_{i}^{p} C_{i, k+1}^{p}\right\} \hat{x}_{i, k+1 \mid k}\right] .
\end{aligned}
$$

To proceed, let $\tilde{x}_{i, k+1 \mid k} \triangleq x_{i, k+1}-\hat{x}_{i, k+1 \mid k}$ be the prediction error, $\tilde{x}_{i, k+1 \mid k+1} \triangleq x_{i, k+1}-\hat{x}_{i, k+1 \mid k+1}$ be the SE error and $\tilde{d}_{i, k+1} \triangleq d_{i, k+1}-\hat{d}_{i, k+1}$ be the estimation error of the unknown input. Then, in view of (1) and (8), we obtain

$$
\begin{aligned}
\tilde{x}_{i, k+1 \mid k}= & \tilde{f}_{k}\left(\tilde{x}_{i, k \mid k}\right)+\sum_{j=1}^{n} \omega_{i j} \Upsilon \tilde{x}_{j, k \mid k}+A_{i, k} \tilde{d}_{i, k} \\
& +B_{i, k} w_{k}, \\
\tilde{x}_{i, k+1 \mid k+1}= & \left(I-K_{i, k+1} \overline{\mathcal{C}}_{i, k+1}\right) \tilde{x}_{i, k+1 \mid k}+K_{i, k+1} \psi_{i, k+1} \\
& -K_{i, k+1} \mathcal{C}_{i, k+1} x_{i, k+1}-K_{i, k+1} F_{i, k+1} \\
& \times \tilde{d}_{i, k+1}-K_{i, k+1} D_{i, k+1} v_{k+1} \\
\tilde{d}_{i, k+1}= & \left(I-G_{i, k+1} F_{i, k+1}\right) d_{i, k+1}+G_{i, k+1} \psi_{i, k+1} \\
& -G_{i, k+1} D_{i, k+1} v_{k+1}-G_{i, k+1} \mathcal{C}_{i, k+1} \\
& \times x_{i, k+1}-G_{i, k+1} \overline{\mathcal{C}}_{i, k+1} \tilde{x}_{i, k+1 \mid k}
\end{aligned}
$$

where

$$
\begin{aligned}
& \tilde{f}_{k}\left(\tilde{x}_{i, k \mid k}\right) \triangleq f_{k}\left(x_{i, k}\right)-f_{k}\left(\hat{x}_{i, k \mid k}\right), \\
& \hbar_{i, k+1}^{1} \triangleq \pi_{i, k+1}^{1}-\bar{\pi}_{i}^{1}, \quad \bar{\hbar}_{i}^{1} \triangleq \bar{\pi}_{i}^{1}, \\
& \hbar_{i, k+1}^{p} \triangleq \prod_{q=1}^{p-1}\left(1-\pi_{i, k+1}^{q}\right) \pi_{i, k+1}^{p}-\prod_{q=1}^{p-1}\left(1-\bar{\pi}_{i}^{q}\right) \bar{\pi}_{i}^{p}, \\
& \overline{\mathcal{C}}_{i, k+1} \triangleq \sum_{p=1}^{z} \bar{\hbar}_{i}^{p} C_{i, k+1}^{p}, \mathcal{C}_{i, k+1} \triangleq \sum_{p=1}^{z} \hbar_{i, k+1}^{p} C_{i, k+1}^{p},
\end{aligned}
$$




$$
\bar{\hbar}_{i}^{p} \triangleq \prod_{q=1}^{p-1}\left(1-\bar{\pi}_{i}^{q}\right) \bar{\pi}_{i}^{p}, \quad p=2,3, \ldots z .
$$

If the following constraint

$$
G_{i, k+1} F_{i, k+1}=I
$$

holds, then the estimation error dynamics of the unknown input reduces to

$$
\begin{aligned}
\tilde{d}_{i, k+1}= & G_{i, k+1} \psi_{i, k+1}-G_{i, k+1} D_{i, k+1} v_{k+1}-G_{i, k+1} \\
& \times \mathcal{C}_{i, k+1} x_{i, k+1}-G_{i, k+1} \overline{\mathcal{C}}_{i, k+1} \tilde{x}_{i, k+1 \mid k} .
\end{aligned}
$$

Remark 4: The condition $\mathrm{R}\left(F_{i, k+1}\right)=n_{d}$ imposed on $F_{i, k+1}$ can guarantee that there exists a matrix $G_{i, k+1}$ such that (10) is satisfied. Note that the constraint (10) is required to be met so as to eliminate the influence of the unknown input in (9). Such kind of constraints has been commonly utilized in the literature, see e.g. [14].

The major objective of this paper is to construct the state and input estimators of form (7) such that the following requirements are simultaneously satisfied.

1) The SE error covariance

$$
P_{i, k+1 \mid k+1}^{x} \triangleq \mathbb{E}\left\{\tilde{x}_{i, k+1 \mid k+1} \tilde{x}_{i, k+1 \mid k+1}^{T}\right\}
$$

and the input estimation error covariance

$$
P_{i, k+1}^{d} \triangleq \mathbb{E}\left\{\tilde{d}_{i, k+1} \tilde{d}_{i, k+1}^{T}\right\}
$$

have upper bounds in the presence of both dynamic ETMs and redundant channels.

2) The estimator gains are parameterized to minimize the obtained upper bounds at every time step.

\section{Main Results}

In this section, we will first derive the upper bounds of the error covariances for both the state and input estimation, and the gain matrices $K_{i, k}$ and $G_{i, k}$ are then parameterized to minimize such upper bounds at time instant $k$.

To proceed, the following two lemmas are given which are helpful for further theoretical developments.

Lemma 1: For any matrices $\mathrm{H}_{1}$ and $\mathrm{H}_{2}$ of compatible dimensions, the following inequality

$$
\mathrm{H}_{1} \mathrm{H}_{2}^{T}+\mathrm{H}_{2} \mathrm{H}_{1}^{T} \leq \mathrm{aH}_{1} \mathrm{H}_{1}^{T}+\mathrm{a}^{-1} \mathrm{H}_{2} \mathrm{H}_{2}^{T}
$$

holds for any scalar a $>0$.

Lemma 2: Let the positive scalars $a_{i, k}$ and $b_{i, k}$ ( $i=$ $1,2, \ldots, n)$ be given. Assume that there exists a set of matrix sequence $\overline{\mathcal{Y}}_{i, k}$ satisfying

$$
\begin{aligned}
\overline{\mathcal{Y}}_{i, k+1} \triangleq & {\left[\left(1+a_{i, k}\right)\left(1+b_{i, k}\right) \gamma_{i}^{2}+\left(1+\mu_{i}\right)\right.} \\
& \left.\times\left(1+a_{i, k}^{-1}\right) / \mu_{i}^{2}\right] \overline{\mathcal{Y}}_{i, k}+\left[\left(1+a_{i, k}\right)\left(1+b_{i, k}^{-1}\right)\right. \\
& \left.+\left(1+a_{i, k}^{-1}\right)\left(1+\mu_{i}^{-1}\right)\right] \sigma_{i}^{2}
\end{aligned}
$$

with initial condition $\overline{\mathcal{Y}}_{i, 0}=\left(\xi_{0}^{i}\right)^{2}$. Then, an upper bound of $\mathcal{Y}_{i, k}$ is $\overline{\mathcal{Y}}_{i, k}$, where $\mathcal{Y}_{i, k} \triangleq \mathbb{E}\left\{\xi_{i, k}^{2}\right\}$.
Proof: By using Lemma 1, one has

$$
\begin{aligned}
\psi_{i, k}^{T} \psi_{i, k} & \leq\left(\frac{1}{\mu_{i}} \xi_{i, k}+\sigma_{i}\right)^{2} \\
& \leq\left(1+\mu_{i}\right) \xi_{i, k}^{2} / \mu_{i}^{2}+\left(1+\mu_{i}{ }^{-1}\right) \sigma_{i}^{2} .
\end{aligned}
$$

Then, according to the analysis in [20], it is easy to obtain that $\mathcal{Y}_{i, k} \leq \overline{\mathcal{Y}}_{i, k}$.

In the following theorem, for state and unknown input estimation, certain upper bounds on the error covariances are obtained, respectively.

Theorem 1: For given positive scalars $c_{u i, k+1}, r_{u i, k+1}$ and $e_{v i, k+1}(u=1,2,3,4 ; v=1,2,3)$, assume that there exist two sets of matrix sequences $\bar{P}_{i, k+1 \mid k+1}^{x}$ and $\bar{P}_{i, k+1}^{d}$ satisfying

$$
\begin{aligned}
& \bar{P}_{i, k+1 \mid k+1}^{x} \\
\triangleq & \Xi_{k+1}^{i}\left(\bar{P}_{i, k+1 \mid k}^{x}, \bar{P}_{i, k+1}^{d}\right) \\
= & \left(1+c_{2 i, k+1}\right)\left(1+e_{2 i, k+1}\right)\left(I-K_{i, k+1} \overline{\mathcal{C}}_{i, k+1}\right) \bar{P}_{i, k+1 \mid k}^{x} \\
& \times\left(I-K_{i, k+1} \overline{\mathcal{C}}_{i, k+1}\right)^{T}+\left(1+c_{2 i, k+1}\right)\left(1+e_{2 i, k+1}^{-1}\right) \\
& \times\left[\left(1+\mu_{i}\right) \overline{\mathcal{Y}}_{i, k+1} / \mu_{i}^{2}+\left(1+\mu_{i}^{-1}\right) \sigma_{i}^{2}\right] K_{i, k+1} K_{i, k+1}^{T} \\
& +\left(1+c_{2 i, k+1}^{-1}\right)\left(1+c_{3 i, k+1}\right)\left(1+r_{3 i, k+1}\right) \sum_{p=1}^{z} \sum_{h=1}^{z} \bar{h}_{i}^{p h} \\
& \times K_{i, k+1} C_{i, k+1}^{p} \hat{x}_{i, k+1 \mid k} \hat{x}_{i, k+1 \mid k}^{T}\left(K_{i, k+1} C_{i, k+1}^{h}\right)^{T} \\
& +\left(1+c_{2 i, k+1}^{-1}\right)\left(1+c_{3 i, k+1}\right)\left(1+r_{3 i, k+1}^{-1}\right) \sum_{p=1}^{z} \sum_{h=1}^{z} \bar{h}_{i}^{p h} \\
& \times K_{i, k+1} C_{i, k+1}^{p} \bar{P}_{i, k+1 \mid k}^{x}\left(K_{i, k+1} C_{i, k+1}^{h}\right)^{T}+\left[r_{2 i, k+1}^{-1}\right. \\
& \left.+\left(1+c_{2 i, k+1}^{-1}\right)\left(1+c_{3 i, k+1}^{-1}\right)\right] K_{i, k+1} F_{i, k+1} \bar{P}_{i, k+1}^{d} \\
& \times F_{i, k+1}^{T} K_{i, k+1}^{T}+\left(1+r_{2 i, k+1}-2 \varrho_{i, k+1}\right) \\
& \times K_{i, k+1} D_{i, k+1} Q_{k+1} D_{i, k+1}^{T} K_{i, k+1}^{T}
\end{aligned}
$$

and

$$
\begin{aligned}
& \bar{P}_{i, k+1}^{d} \\
= & \left(1+c_{4 i, k+1}\right)\left(1+r_{4 i, k+1}\right) G_{i, k+1} \overline{\mathcal{C}}_{i, k+1} \bar{P}_{i, k+1 \mid k}^{x} \overline{\mathcal{C}}_{i, k+1}^{T} \\
& \times G_{i, k+1}^{T}+\left[\left(1+\mu_{i}\right) \overline{\mathcal{Y}}_{i, k+1} / \mu_{i}^{2}+\left(1+\mu_{i}{ }^{-1}\right) \sigma_{i}^{2}\right] \\
& \times\left(1+c_{4 i, k+1}\right)\left(1+r_{4 i, k+1}^{-1}\right) G_{i, k+1} G_{i, k+1}^{T}+\left(1+c_{4 i, k+1}^{-1}\right) \\
& \times\left(1+e_{3 i, k+1}\right) \sum_{p=1}^{z} \sum_{h=1}^{z} \bar{\hbar}_{i}^{p h} G_{i, k+1} C_{i, k+1}^{p} \hat{x}_{i, k+1 \mid k} \hat{x}_{i, k+1 \mid k}^{T} \\
& \times\left(G_{i, k+1} C_{i, k+1}^{h}\right)^{T}+\left(1+c_{4 i, k+1}^{-1}\right)\left(1+e_{3 i, k+1}^{-1}\right) \\
& \times \sum_{p=1}^{z} \sum_{h=1}^{z} \bar{\hbar}_{i}^{p h} G_{i, k+1} C_{i, k+1}^{p} \bar{P}_{i, k+1 \mid k}^{x}\left(G_{i, k+1} C_{i, k+1}^{h}\right)^{T} \\
& +\left(1-2 \varrho_{i, k+1}\right) G_{i, k+1} D_{i, k+1} Q_{k+1} D_{i, k+1}^{T} G_{i, k+1}^{T}
\end{aligned}
$$

with initial conditions $\bar{P}_{i, 0}^{x}=P_{i, 0}^{x}$ and $\bar{P}_{i, 0}^{d}=\bar{d}_{i}^{2}$ and

$$
\begin{aligned}
\bar{P}_{i, k+1 \mid k}^{x} \triangleq & \left(1+c_{1 i, k}\right)\left(1+r_{1 i, k}\right) \ell_{k}^{2} \operatorname{tr}\left\{\bar{P}_{i, k \mid k}^{x}\right\} I+\left(1+c_{1 i, k}\right) \\
& \times\left(1+r_{1 i, k}^{-1}\right) E_{k} \bar{P}_{i, k \mid k}^{x} E_{k}^{T}+\left(1+c_{i, k}^{-1}\right)\left(1+e_{1 i, k}\right) \\
& \times \bar{\omega}_{i} \sum_{j=1}^{n}\left|\omega_{i j}\right| \Upsilon \bar{P}_{j, k \mid k} \Upsilon^{T}+\left(1+c_{1 i, k}^{-1}\right) \\
& \times\left(1+e_{i, k}^{-1}\right) A_{i, k} \bar{P}_{i, k}^{d} A_{i, k}^{T}+B_{i, k} R_{k} B_{i, k}^{T},
\end{aligned}
$$




$$
\bar{\omega}_{i} \triangleq \sum_{j=1}^{n}\left|\omega_{i j}\right|, \quad \bar{\hbar}_{i}^{p h} \triangleq\left\{\begin{array}{cc}
\bar{\hbar}_{i}^{p}\left(1-\bar{\hbar}_{i}^{p}\right), & p=h, \\
-\bar{\hbar}_{i}^{p} \bar{\hbar}_{i}^{h}, & p \neq h,
\end{array}\right.
$$

$\varrho_{i, k+1} \triangleq \begin{cases}0, & \text { the condition in }(5) \text { is satisfied, } \\ 1, & \text { otherwise. }\end{cases}$

Then, $\bar{P}_{i, k+1 \mid k+1}^{x}$ and $\bar{P}_{i, k+1}^{d}$ are, respectively, the upper bounds on the estimation error covariances $P_{i, k+1 \mid k+1}^{x}$ and $P_{i, k+1}^{d}$, i.e.,

$$
P_{i, k+1 \mid k+1}^{x} \leq \bar{P}_{i, k+1 \mid k+1}^{x}, \quad P_{i, k+1}^{d} \leq \bar{P}_{i, k+1}^{d} .
$$

Proof: This proof is conducted via mathematical induction. Assume that $P_{i, k \mid k}^{x} \leq \bar{P}_{i, k \mid k}^{x}$ and $P_{i, k}^{d} \leq \bar{P}_{i, k}^{d}$ are true. Then, we need to prove that $P_{i, k+1 \mid k+1}^{x} \leq \bar{P}_{i, k+1 \mid k+1}^{x}$ and $P_{i, k+1}^{d} \leq \bar{P}_{i, k+1}^{d}$.

First, since $P_{i, k+1 \mid k}^{x}$ is defined as the prediction error covariance, it follows from Lemma 1 and (9) that

$$
\begin{aligned}
& P_{i, k+1 \mid k}^{x} \triangleq \mathbb{E}\left\{\tilde{x}_{i, k+1 \mid k} \tilde{x}_{i, k+1 \mid k}^{T}\right\} \\
= & \mathbb{E}\left\{\left(\tilde{f}_{k}\left(\tilde{x}_{i, k \mid k}\right)+\sum_{j=1}^{n} \omega_{i j} \Upsilon \tilde{x}_{j, k \mid k}+A_{i, k} \tilde{d}_{i, k}\right.\right. \\
& \left.+B_{i, k} w_{k}\right)\left(\tilde{f}_{k}\left(\tilde{x}_{i, k \mid k}\right)+\sum_{j=1}^{n} \omega_{i j} \Upsilon \tilde{x}_{j, k \mid k}\right. \\
& \left.\left.+A_{i, k} \tilde{d}_{i, k}+B_{i, k} w_{k}\right)^{T}\right\} \\
\leq & \mathbb{E}\left\{\left(1+c_{1 i, k}\right)\left(1+r_{1 i, k}\right)\left\|\tilde{f}_{k}\left(\tilde{x}_{i, k \mid k}\right)-E_{k} \tilde{x}_{i, k \mid k}\right\|^{2} I\right. \\
& +\left(1+c_{1 i, k}\right)\left(1+r_{1 i, k}^{-1}\right) E_{k} \tilde{x}_{i, k \mid k} \tilde{x}_{i, k \mid k}^{T} E_{k}^{T} \\
& +\left(1+c_{1 i, k}^{-1}\right)\left(1+e_{1 i, k}\right) \bar{\omega}_{i} \sum_{j=1}^{n}\left|\omega_{i j}\right| \Upsilon P_{j, k \mid k}^{x} \Upsilon^{T} \\
& \left.+\left(1+c_{1 i, k}^{-1}\right)\left(1+e_{1 i, k}^{-1}\right) A_{i, k} \tilde{d}_{i, k} \tilde{d}_{i, k}^{T} A_{i, k}^{T}\right\} \\
& +B_{i, k} R_{k} B_{i, k}^{T},
\end{aligned}
$$

where $c_{1 i, k}, r_{1 i, k}$ and $e_{1 i, k}$ are positive scalars. From Assumption 1 , we further have

$$
\begin{aligned}
& P_{i, k+1 \mid k}^{x} \\
\leq & \left(1+c_{1 i, k}\right)\left(1+r_{1 i, k}\right) \ell_{k}^{2} \operatorname{tr}\left\{P_{i, k \mid k}^{x}\right\} I+\left(1+c_{1 i, k}\right) \\
& \times\left(1+r_{1 i, k}^{-1}\right) E_{k} P_{i, k \mid k}^{x} E_{k}^{T}+\left(1+c_{1 i, k}^{-1}\right)\left(1+e_{1 i, k}\right) \\
& \times \bar{\omega}_{i} \sum_{j=1}^{n}\left|\omega_{i j}\right| \Upsilon P_{j, k \mid k}^{x} \Upsilon^{T}+\left(1+c_{1 i, k}^{-1}\right) \\
& \times\left(1+e_{1 i, k}^{-1}\right) A_{i, k} P_{i, k \mid k}^{d} A_{i, k}^{T}+B_{i, k} R_{k} B_{i, k}^{T} .
\end{aligned}
$$

Based on $P_{i, k \mid k}^{x} \leq \bar{P}_{i, k \mid k}^{x}$ and $P_{i, k \mid k}^{d} \leq \bar{P}_{i, k \mid k}^{d}$, one has

$$
P_{i, k+1 \mid k}^{x} \leq \bar{P}_{i, k+1 \mid k}^{x} .
$$

From (9), the covariance of the SE error $P_{i, k+1 \mid k+1}^{x}$ is calculated as

$$
\begin{aligned}
& P_{i, k+1 \mid k+1}^{x} \\
= & \mathbb{E}\left\{\left[\left(I-K_{i, k+1} \overline{\mathcal{C}}_{i, k+1}\right) \tilde{x}_{i, k+1 \mid k}+K_{i, k+1} \psi_{i, k+1}\right.\right. \\
& -K_{i, k+1} \mathcal{C}_{i, k+1} x_{i, k+1}-K_{i, k+1} F_{i, k+1} \tilde{d}_{i, k+1} \\
& \left.-K_{i, k+1} D_{i, k+1} v_{k+1}\right]\left[\left(I-K_{i, k+1} \overline{\mathcal{C}}_{i, k+1}\right) \tilde{x}_{i, k+1 \mid k}\right.
\end{aligned}
$$

$$
\begin{aligned}
& +K_{i, k+1} \psi_{i, k+1}-K_{i, k+1} \mathcal{C}_{i, k+1} x_{i, k+1} \\
& \left.\left.-K_{i, k+1} F_{i, k+1} \tilde{d}_{i, k+1}-K_{i, k+1} D_{i, k+1} v_{k+1}\right]^{T}\right\} \\
= & \mathbb{E}\left\{\left[\left(I-K_{i, k+1} \overline{\mathcal{C}}_{i, k+1}\right) \tilde{x}_{i, k+1 \mid k}+K_{i, k+1} \psi_{i, k+1}\right.\right. \\
& \left.-K_{i, k+1} \mathcal{C}_{i, k+1} x_{i, k+1}-K_{i, k+1} F_{i, k+1} \tilde{d}_{i, k+1}\right] \\
& \times\left[\left(I-K_{i, k+1} \overline{\mathcal{C}}_{i, k+1}\right) \tilde{x}_{i, k+1 \mid k}+K_{i, k+1} \psi_{i, k+1}\right. \\
& \left.\left.-K_{i, k+1} \mathcal{C}_{i, k+1} x_{i, k+1}-K_{i, k+1} F_{i, k+1} \tilde{d}_{i, k+1}\right]^{T}\right\} \\
& +K_{i, k+1} D_{i, k+1} \mathbb{E}\left\{v_{k+1} v_{k+1}^{T}\right\} D_{i, k+1}^{T} \\
& \times K_{i, k+1}^{T}+\mathfrak{E}_{i, k+1}+\mathfrak{E}_{i, k+1}^{T}+\mathfrak{H}_{i, k+1}+\mathfrak{H}_{i, k+1}^{T},
\end{aligned}
$$

where

$$
\begin{aligned}
& \mathfrak{E}_{i, k+1} \triangleq-\mathbb{E}\left\{K_{i, k+1} \psi_{i, k+1} v_{k+1}^{T} D_{i, k+1}^{T} K_{i, k+1}^{T}\right\}, \\
& \mathfrak{H}_{i, k+1} \triangleq \mathbb{E}\left\{K_{i, k+1} D_{i, k+1} v_{k+1} \tilde{d}_{i, k+1}^{T} F_{i, k+1}^{T} K_{i, k+1}^{T}\right\} .
\end{aligned}
$$

With the help of Lemma 1, we have

$$
\begin{aligned}
& P_{i, k+1 \mid k+1}^{x} \\
& \leq\left(1+c_{2 i, k+1}\right) \mathbb{E}\left\{\left[\left(I-K_{i, k+1} \overline{\mathcal{C}}_{i, k+1}\right) \tilde{x}_{i, k+1 \mid k}\right.\right. \\
&\left.+K_{i, k+1} \psi_{i, k+1}\right]\left[\left(I-K_{i, k+1} \overline{\mathcal{C}}_{i, k+1}\right) \tilde{x}_{i, k+1 \mid k}\right. \\
&\left.\left.+K_{i, k+1} \psi_{i, k+1}\right]^{T}\right\}+\left(1+c_{2 i, k+1}^{-1}\right) \mathbb{E}\left\{\left(K_{i, k+1} \mathcal{C}_{i, k+1}\right.\right. \\
&\left.\times x_{i, k+1}+K_{i, k+1} F_{i, k+1} \tilde{d}_{i, k+1}\right)\left(K_{i, k+1} \mathcal{C}_{i, k+1} x_{i, k+1}\right. \\
&\left.\left.+K_{i, k+1} F_{i, k+1} \tilde{d}_{i, k+1}\right)^{T}\right\}+\left(1+r_{2 i, k+1}\right) K_{i, k+1} \\
& \times D_{i, k+1} \mathbb{E}\left\{v_{k+1} v_{k+1}^{T}\right\} D_{i, k+1}^{T} K_{i, k+1}^{T} \\
&+r_{2 i, k+1}^{-1} K_{i, k+1} F_{i, k+1} \mathbb{E}\left\{\tilde{d}_{i, k+1} \tilde{d}_{i, k+1}^{T}\right\} \\
& \times F_{i, k+1}^{T} K_{i, k+1}^{T}+\mathfrak{E}_{i, k+1}+\mathfrak{E}_{i, k+1}^{T} \\
& \leq\left(1+c_{2 i, k+1}\right)\left(1+e_{2 i, k+1}^{-1}\right) K_{i, k+1} \mathbb{E}\left\{\psi_{i, k+1} \psi_{i, k+1}^{T}\right\} K_{i, k+1}^{T} \\
&+\left(1+c_{2 i, k+1}^{-1}\right)\left(1+c_{3 i, k+1}\right) \mathbb{E}\left\{K_{i, k+1} \mathcal{C}_{i, k+1} x_{i, k+1} x_{i, k+1}^{T}\right. \\
&\left.\times \mathcal{C}_{i, k+1}^{T} K_{i, k+1}^{T}\right\}+\left(1+c_{2 i, k+1}\right)\left(1+e_{2 i, k+1}\right)\left(I-K_{i, k+1}\right. \\
&\left.\times \overline{\mathcal{C}}_{i, k+1}\right) \mathbb{E}\left\{\tilde{x}_{i, k+1 \mid k} \tilde{x}_{i, k+1 \mid k}^{T}\right\}\left(I-K_{i, k+1} \overline{\mathcal{C}}_{i, k+1}\right)^{T} \\
&+\left[\left(1+c_{2 i, k+1}^{-1}\right)\left(1+c_{3 i, k+1}^{-1}\right)+r_{2 i, k+1}^{-1}\right] K_{i, k+1} F_{i, k+1} \\
& \times \mathbb{E}\left\{\tilde{d}_{i, k+1} \tilde{d}_{i, k+1}^{T}\right\} F_{i, k+1}^{T} K_{i, k+1}^{T}+\left(1+r_{2 i, k+1}\right) K_{i, k+1} \\
& \times D_{i, k+1} Q_{k+1} D_{i, k+1}^{T} K_{i, k+1}^{T}+\mathfrak{E}_{i, k+1}+\mathfrak{E}_{i, k+1}^{T}
\end{aligned}
$$

with $c_{2 i, k+1}, c_{3 i, k+1}, e_{2 i, k+1}$ and $r_{2 i, k+1}$ being positive scalars.

Since $\mathbb{E}\left\{\left(\hbar_{i, k+1}^{p}\right)^{2}\right\}=\bar{\hbar}_{i}^{p}\left(1-\bar{\hbar}_{i}^{p}\right)$ and $\mathbb{E}\left\{\hbar_{i, k+1}^{p} \hbar_{i, k+1}^{h}\right\}=$ $-\bar{\hbar}_{i}^{p} \bar{\hbar}_{i}^{h}(p \neq h)$, it is obtained that

$$
\begin{aligned}
& \mathbb{E}\left\{K_{i, k+1} \mathcal{C}_{i, k+1} x_{i, k+1} x_{i, k+1}^{T} \mathcal{C}_{i, k+1}^{T} K_{i, k+1}^{T}\right\} \\
= & \sum_{p=1}^{z} \bar{\hbar}_{i}^{p}\left(1-\bar{\hbar}_{i}^{p}\right) K_{i, k+1} C_{i, k+1}^{p} \mathbb{E}\left\{x_{i, k+1} x_{i, k+1}^{T}\right\} \\
& \times\left(K_{i, k+1} C_{i, k+1}^{p}\right)^{T}-\sum_{1 \leq p, h \leq z, p \neq h} \bar{\hbar}_{i}^{p} \bar{\hbar}_{i}^{h} K_{i, k+1}
\end{aligned}
$$




$$
\times C_{i, k+1}^{p} \mathbb{E}\left\{x_{i, k+1} x_{i, k+1}^{T}\right\}\left(K_{i, k+1} C_{i, k+1}^{h}\right)^{T} .
$$

Next, it follows from Lemma 1 that

$$
\begin{aligned}
& \mathbb{E}\left\{x_{i, k+1} x_{i, k+1}^{T}\right\} \\
= & \mathbb{E}\left\{\left(\hat{x}_{i, k+1}+\tilde{x}_{i, k+1 \mid k}^{T}\right)\left(\hat{x}_{i, k+1}+\tilde{x}_{i, k+1 \mid k}^{T}\right)^{T}\right\} \\
\leq & \left(1+r_{3 i, k+1}\right) \hat{x}_{i, k+1 \mid k} \hat{x}_{i, k+1 \mid k}^{T}+\left(1+r_{3 i, k+1}^{-1}\right) P_{i, k+1 \mid k}^{x},
\end{aligned}
$$

where $r_{3 i, k+1}$ are positive scalars. that

Recalling the definitions of $\psi_{i, k+1}$ and $\varrho_{i, k+1}$, it is obvious

$$
\begin{aligned}
\mathbb{E}\left\{\psi_{i, k+1} v_{k+1}^{T}\right\} & =\mathbb{E}\left\{\left(y_{i, k+1}-y_{i, \kappa_{l}^{i}}\right) v_{k+1}^{T}\right\} \\
& =\varrho_{i, k+1} D_{i, k+1} Q_{k+1}
\end{aligned}
$$

holds for all $k+1 \in\left[\kappa_{l}^{i}, \kappa_{l+1}^{i}\right)$. Hence,

$$
\mathfrak{E}_{i, k+1}=-\varrho_{i, k+1} K_{i, k+1} D_{i, k+1} Q_{k+1} D_{i, k+1}^{T} K_{i, k+1}^{T} .
$$

In addition, it is not difficult to see that

$$
\psi_{i, k+1} \psi_{i, k+1}^{T} \leq \psi_{i, k+1}^{T} \psi_{i, k+1} I
$$

always holds. Then, it is known from (13) that

$$
\begin{aligned}
& \mathbb{E}\left\{\psi_{i, k+1} \psi_{i, k+1}^{T}\right\} \\
\leq & {\left[\left(1+\mu_{i}\right) \overline{\mathcal{Y}}_{i, k+1} / \mu_{i}^{2}+\left(1+\mu_{i}{ }^{-1}\right) \sigma_{i}^{2}\right] I . }
\end{aligned}
$$

Substituting (22)-(23), (25) and (27) into (21) leads to

$$
\begin{aligned}
& P_{i, k+1 \mid k+1}^{x} \\
\leq & \left(1+c_{2 i, k+1}\right)\left(1+e_{2 i, k+1}\right)\left(I-K_{i, k+1} \overline{\mathcal{C}}_{i, k+1}\right) P_{i, k+1 \mid k}^{x} \\
& \times\left(I-K_{i, k+1} \overline{\mathcal{C}}_{i, k+1}\right)^{T}+\left(1+c_{2 i, k+1}\right)\left(1+e_{2 i, k+1}^{-1}\right) \\
& \times\left[\left(1+\mu_{i}\right) \overline{\mathcal{Y}}_{i, k+1} / \mu_{i}^{2}+\left(1+\mu_{i}{ }^{-1}\right) \sigma_{i}^{2}\right] K_{i, k+1} K_{i, k+1}^{T} \\
& +\left(1+c_{2 i, k+1}^{-1}\right)\left(1+c_{3 i, k+1}\right)\left(1+r_{3 i, k+1}\right) \sum_{p=1}^{z} \sum_{h=1}^{z} \bar{h}_{i}^{p h} \\
& \times K_{i, k+1} C_{i, k+1}^{p} \hat{x}_{i, k+1 \mid k} \hat{x}_{i, k+1 \mid k}^{T}\left(K_{i, k+1} C_{i, k+1}^{h}\right)^{T} \\
& +\left(1+c_{2 i, k+1}^{-1}\right)\left(1+c_{3 i, k+1}\right)\left(1+r_{3 i, k+1}^{-1}\right) \sum_{p=1}^{z} \sum_{h=1}^{z} \bar{\hbar}_{i}^{p h} \\
& \times K_{i, k+1} C_{i, k+1}^{p} P_{i, k+1 \mid k}^{x}\left(K_{i, k+1} C_{i, k+1}^{h}\right)^{T}+\left[r_{2 i, k+1}^{-1}\right. \\
& \left.+\left(1+c_{2 i, k+1}^{-1}\right)\left(1+c_{3 i, k+1}^{-1}\right)\right] K_{i, k+1} F_{i, k+1} P_{i, k+1}^{d} \\
& \times F_{i, k+1}^{T} K_{i, k+1}^{T}+\left(1+r_{2 i, k+1}-2 \varrho_{i, k+1}\right) K_{i, k+1} \\
& \times D_{i, k+1} Q_{k+1} D_{i, k+1}^{T} K_{i, k+1}^{T}
\end{aligned}
$$

which, together with (19), implies

$$
P_{i, k+1 \mid k+1}^{x} \leq \Xi_{k+1}^{i}\left(\bar{P}_{i, k+1 \mid k}^{x}, P_{i, k+1}^{d}\right) .
$$

Subsequently, we are in a position to show that $P_{i, k+1}^{d} \leq$ $\bar{P}_{i, k+1}^{d}$. From (11), the input estimation error covariance is deduced as follows:

$$
\begin{aligned}
& P_{i, k+1}^{d}=\mathbb{E}\left\{\tilde{d}_{i, k+1} \tilde{d}_{i, k+1}^{T}\right\} \\
= & \mathbb{E}\left\{\left(G_{i, k+1} \psi_{i, k+1}-G_{i, k+1} \mathcal{C}_{i, k+1} x_{i, k+1}\right.\right. \\
& \left.-G_{i, k+1} \overline{\mathcal{C}}_{i, k+1} \tilde{x}_{i, k+1 \mid k}-G_{i, k+1} D_{i, k+1} v_{k+1}\right)
\end{aligned}
$$

$$
\begin{aligned}
& \times\left(G_{i, k+1} \psi_{i, k+1}-G_{i, k+1} D_{i, k+1} v_{k+1}-G_{i, k+1}\right. \\
& \left.\left.\times \mathcal{C}_{i, k+1} x_{i, k+1}-G_{i, k+1} \overline{\mathcal{C}}_{i, k+1} \tilde{x}_{i, k+1 \mid k}\right)^{T}\right\} \\
= & \mathbb{E}\left\{\left(G_{i, k+1} \psi_{i, k+1}-G_{i, k+1} \mathcal{C}_{i, k+1} x_{i, k+1}\right.\right. \\
& \left.-G_{i, k+1} \overline{\mathcal{C}}_{i, k+1} \tilde{x}_{i, k+1 \mid k}\right)\left(G_{i, k+1} \psi_{i, k+1}\right. \\
& \left.\left.-G_{i, k+1} \mathcal{C}_{i, k+1} x_{i, k+1}-G_{i, k+1} \overline{\mathcal{C}}_{i, k+1} \tilde{x}_{i, k+1 \mid k}\right)^{T}\right\} \\
& +G_{i, k+1} D_{i, k+1} \mathbb{E}\left\{v_{k+1} v_{k+1}^{T}\right\} D_{i, k+1}^{T} G_{i, k+1}^{T} \\
& +\mathfrak{J}_{i, k+1}+\mathfrak{J}_{i, k+1}^{T},
\end{aligned}
$$

where

$$
\mathfrak{J}_{i, k+1} \triangleq-G_{i, k+1} \mathbb{E}\left\{\psi_{i, k+1} v_{k+1}^{T}\right\} D_{i, k+1}^{T} G_{i, k+1}^{T} .
$$

Repeating the derivation process as in (20)-(29), one obtains

$$
\begin{aligned}
& P_{i, k+1}^{d} \\
\leq & \left(1+c_{4 i, k+1}\right)\left(1+r_{4 i, k+1}\right) G_{i, k+1} \overline{\mathcal{C}}_{i, k+1} \bar{P}_{i, k+1 \mid k}^{x} \overline{\mathcal{C}}_{i, k+1}^{T} \\
& \times G_{i, k+1}^{T}+\left[\left(1+\mu_{i}\right) \overline{\mathcal{Y}}_{i, k+1} / \mu_{i}^{2}+\left(1+\mu_{i}{ }^{-1}\right) \sigma_{i}^{2}\right] \\
& \times\left(1+c_{4 i, k+1}\right)\left(1+r_{4 i, k+1}^{-1}\right) G_{i, k+1} G_{i, k+1}^{T}+\left(1+c_{4 i, k+1}^{-1}\right) \\
& \times\left(1+e_{3 i, k+1}\right) \sum_{p=1}^{z} \sum_{h=1}^{z} \bar{\hbar}_{i}^{p h} G_{i, k+1} C_{i, k+1}^{p} \hat{x}_{i, k+1 \mid k} \hat{x}_{i, k+1 \mid k}^{T} \\
& \times\left(G_{i, k+1} C_{i, k+1}^{h}\right)^{T}+\left(1+c_{4 i, k+1}^{-1}\right)\left(1+e_{3 i, k+1}^{-1}\right) \\
& \times \sum_{p=1}^{z} \sum_{h=1}^{z} \bar{\hbar}_{i}^{p h} G_{i, k+1} C_{i, k+1}^{p} \bar{P}_{i, k+1 \mid k}^{x}\left(G_{i, k+1} C_{i, k+1}^{h}\right)^{T} \\
& +\left(1-2 \varrho_{i, k+1}\right) G_{i, k+1} D_{i, k+1} Q_{k+1} D_{i, k+1}^{T} G_{i, k+1}^{T} \\
= & \bar{P}_{i, k+1}^{d}
\end{aligned}
$$

with $c_{4 i, k+1}, r_{4 i, k+1}$ and $e_{3 i, k+1}$ being positive scalars, which further indicates

$$
P_{i, k+1 \mid k+1}^{x} \leq \bar{P}_{i, k+1 \mid k+1}^{x} .
$$

The proof is now complete.

According to the above results, we are now going to minimize the obtained upper bounds $\bar{P}_{i, k+1 \mid k+1}^{x}$ and $\bar{P}_{i, k+1}^{d}$ by designing the estimator gains $K_{i, k+1}$ and $G_{i, k+1}$.

Theorem 2: The upper bounds on the estimation error covariances given by the recursions (14) and (15) can be minimized by adopting the following estimator gains

$$
G_{i, k+1}=\left(F_{i, k+1}^{T} \Omega_{i, k+1}^{-1} F_{i, k+1}\right)^{-1} F_{i, k+1}^{T} \Omega_{i, k+1}^{-1}
$$

and

$$
K_{i, k+1}=\Phi_{i, k+1} \Theta_{i, k+1}^{-1},
$$

where

$$
\begin{aligned}
\Omega_{i, k+1} \triangleq & \left(1+c_{4 i, k+1}\right)\left(1+r_{4 i, k+1}\right) \overline{\mathcal{C}}_{i, k+1} \bar{P}_{i, k+1 \mid k}^{x} \overline{\mathcal{C}}_{i, k+1}^{T} \\
& +\left(1+c_{4 i, k+1}\right)\left(1+r_{4 i, k+1}^{-1}\right)\left[\left(1+\mu_{i}\right) \overline{\mathcal{Y}}_{i, k+1} / \mu_{i}^{2}\right. \\
& \left.+\left(1+\mu_{i}{ }^{-1}\right) \sigma_{i}^{2}\right] I+\left(1+c_{4 i, k+1}^{-1}\right)\left(1+e_{3 i, k+1}\right) \\
& \times \sum_{p=1}^{z} \sum_{h=1}^{z} \bar{\hbar}_{i}^{p h} C_{i, k+1}^{p} \hat{x}_{i, k+1 \mid k} \hat{x}_{i, k+1 \mid k}^{T}\left(C_{i, k+1}^{h}\right)^{T}
\end{aligned}
$$




$$
\begin{aligned}
& +\left(1+c_{4 i, k+1}^{-1}\right)\left(1+e_{3 i, k+1}^{-1}\right) \sum_{p=1}^{z} \sum_{h=1}^{z} \bar{\hbar}_{i}^{p h} C_{i, k+1}^{p} \\
& \times \bar{P}_{i, k+1 \mid k}^{x}\left(C_{i, k+1}^{h}\right)^{T}+\left(1-2 \varrho_{i, k+1}\right) \\
& \times D_{i, k+1} Q_{k+1} D_{i, k+1}^{T}, \\
\Phi_{i, k+1} \triangleq & \left(1+c_{2 i, k+1}\right)\left(1+e_{2 i, k+1}\right) \bar{P}_{i, k+1 \mid k}^{x} \overline{\mathcal{C}}_{i, k+1}^{T}, \\
\Theta_{i, k+1} \triangleq & \left(1+c_{2 i, k+1}\right)\left(1+e_{2 i, k+1}\right) \overline{\mathcal{C}}_{i, k+1} \bar{P}_{i, k+1 \mid k}^{x} \overline{\mathcal{C}}_{i, k+1}^{T} \\
& +\left(1+c_{2 i, k+1}\right)\left(1+e_{2 i, k+1}^{-1}\right)\left[\left(1+\mu_{i}\right) \overline{\mathcal{Y}}_{i, k+1} / \mu_{i}^{2}\right. \\
& \left.+\left(1+\mu_{i}^{-1}\right) \sigma_{i}^{2}\right] I+\left(1+c_{2 i, k+1}^{-1}\right)\left(1+c_{3 i, k+1}\right) \\
& \times\left(1+r_{3 i, k+1}\right) \sum_{p=1}^{z} \sum_{h=1}^{z} \bar{\hbar}_{i}^{p h} C_{i, k+1}^{p} \hat{x}_{i, k+1 \mid k} \hat{x}_{i, k+1 \mid k}^{T} \\
& \times\left(C_{i, k+1}^{h}\right)^{T}+\left(1+c_{2 i, k+1}^{-1}\right)\left(1+c_{3 i, k+1}\right)(1+ \\
& \left.+r_{3 i, k+1}^{-1}\right) \sum_{p=1}^{z} \sum_{h=1}^{z} \bar{\hbar}_{i}^{p h} C_{i, k+1}^{p} \bar{P}_{i, k+1 \mid k}^{x}\left(C_{i, k+1}^{h}\right)^{T} \\
& +\left[r_{2 i, k+1}^{-1}+\left(1+c_{2 i, k+1}^{-1}\right)\left(1+c_{3 i, k+1}^{-1}\right)\right] F_{i, k+1} \\
& \times \bar{P}_{i, k+1}^{d} F_{i, k+1}^{T}+\left(1+r_{2 i, k+1}-2 \varrho_{i, k+1}\right) \\
& \times D_{i, k+1} Q_{k+1} D_{i, k+1}^{T} .
\end{aligned}
$$

Moreover, the minimal upper bounds $\bar{P}_{i, k+1}^{d}$ and $\bar{P}_{i, k+1 \mid k+1}^{x}$ can be given by:

$$
\bar{P}_{i, k+1}^{d}=\left(F_{i, k+1}^{T} \Omega_{i, k+1}^{-1} F_{i, k+1}\right)^{-1}
$$

and

$$
\begin{aligned}
\bar{P}_{i, k+1 \mid k+1}^{x}= & \left(1+c_{2 i, k+1}\right)\left(1+e_{2 i, k+1}\right) \bar{P}_{i, k+1 \mid k}^{x} \\
& -\Phi_{i, k+1} \Theta_{i, k+1}^{-1} \Phi_{i, k+1}^{T} .
\end{aligned}
$$

Proof: From (15), the upper bound $\bar{P}_{i, k+1}^{d}$ can be expressed of the following form:

$$
\bar{P}_{i, k+1}^{d}=G_{i, k+1} \Omega_{i, k+1} G_{i, k+1}^{T} .
$$

It is desirable to design $G_{i, k+1}$ satisfying the constraint (10) such that $\bar{P}_{i, k+1}^{d}$ is minimum. We will utilize the Lagrange multiplier method to solve this issue. A Lagrange function is constructed as follows:

$$
\begin{aligned}
\mathcal{H}_{i, k+1}= & G_{i, k+1} \Omega_{i, k+1} G_{i, k+1}^{T}+\left(G_{i, k+1} F_{i, k+1}-I\right) \\
& \times \Delta_{i, k+1}^{T}+\Delta_{i, k+1}\left(G_{i, k+1} F_{i, k+1}-I\right)^{T}
\end{aligned}
$$

where $\Delta_{i, k+1}$ is the Lagrange factor which is a symmetric matrix with appropriate dimension.

By using the completing-the-square method, (39) can be further obtained as

$$
\begin{aligned}
\mathcal{H}_{i, k+1}= & G_{i, k+1} \Omega_{i, k+1} G_{i, k+1}^{T}+G_{i, k+1} F_{i, k+1} \Delta_{i, k+1}^{T} \\
& -\Delta_{i, k+1}^{T}+\Delta_{i, k+1} F_{i, k+1}^{T} G_{i, k+1}^{T}-\Delta_{i, k+1} \\
= & {\left[G_{i, k+1}-\Delta_{i, k+1}^{T} F_{i, k+1}^{T} \Omega_{i, k+1}^{-1}\right] \Omega_{i, k+1} } \\
& \times\left[G_{i, k+1}-\Delta_{i, k+1}^{T} F_{i, k+1}^{T} \Omega_{i, k+1}^{-1}\right]^{T} \\
& -\left[\Delta_{i, k+1}-\left(F_{i, k+1}^{T} \Omega_{i, k+1}^{-1} F_{i, k+1}\right)^{-1}\right] \\
& \times\left(F_{i, k+1}^{T} \Omega_{i, k+1}^{-1} F_{i, k+1}\right)\left[\Delta_{i, k+1}\right. \\
& \left.-\left(F_{i, k+1}^{T} \Omega_{i, k+1}^{-1} F_{i, k+1}\right)^{-1}\right]^{T} \\
& +\left(F_{i, k+1}^{T} \Omega_{i, k+1}^{-1} F_{i, k+1}\right)^{-1} .
\end{aligned}
$$

In order to achieve minimum $\mathcal{H}_{i, k+1}$, the estimator gain $G_{i, k+1}$ should be given by

$$
G_{i, k+1}=\Delta_{i, k+1}^{T} F_{i, k+1}^{T} \Omega_{i, k+1}^{-1} .
$$

Submitting (41) into the constraint (10) leads to

$$
\Delta_{i, k+1}=\left(F_{i, k+1}^{T} \Omega_{i, k+1}^{-1} F_{i, k+1}\right)^{-1} .
$$

Therefore, it is easy to see that the minimum of the upper bound $\bar{P}_{i, k+1}^{d}$ satisfying constraint (10) can be achieved if (33) holds and the minimal value of $\bar{P}_{i, k+1}^{d}$ is obtained by (36).

Next, bearing in mind the notations in (35), it is obtained from (14) that

$$
\begin{aligned}
& \bar{P}_{i, k+1 \mid k+1}^{x} \\
= & \left(1+c_{2 i, k+1}\right)\left(1+e_{2 i, k+1}\right) \bar{P}_{i, k+1 \mid k}^{x}-K_{i, k+1} \Phi_{i, k+1}^{T} \\
& -\Phi_{i, k+1} K_{i, k+1}^{T}+K_{i, k+1} \Theta_{i, k+1} K_{i, k+1}^{T} \\
= & \left(1+c_{2 i, k+1}\right)\left(1+e_{2 i, k+1}\right) \bar{P}_{i, k+1 \mid k}^{x}-\Phi_{i, k+1} \Theta_{i, k+1}^{-1} \\
& \times \Phi_{i, k+1}^{T}+\left(K_{i, k+1}-\Phi_{i, k+1} \Theta_{i, k+1}^{-1}\right) \Theta_{i, k+1} \\
& \times\left(K_{i, k+1}-\Phi_{i, k+1} \Theta_{i, k+1}^{-1}\right)^{T} .
\end{aligned}
$$

By noting $\Theta_{i, k+1}>0$, it is easy to see that $\bar{P}_{i, k+1 \mid k+1}^{x}$ is minimized by selecting $K_{i, k+1}=\Phi_{i, k+1} \Theta_{i, k+1}^{-1}$, and then the minimum of $\bar{P}_{i, k+1 \mid k+1}^{x}$ is expressed by (37).

Remark 5: So far, we have made one of the first attempts to tackle the simultaneous SUIE problem for time-varying $\mathrm{CNs}$ subject to dynamic ETMs and redundant channels. In Theorem 1, the upper bounds on the error covariances of the state and input estimation have been obtained at each time instant. Subsequently, such obtained upper bounds have been minimized by appropriately constructing the estimators in Theorem 2 via solving two sets of recursions. It is worth mentioning that the analysis method developed in our paper is absolutely applicable in the case that the network topology is not strongly connected and the corresponding results could be derived accordingly. In comparison to the existing literature on $\mathrm{SE}$ problem for time-varying $\mathrm{CNs}$, the major features of the proposed main results lie in the following two aspects: 1) the influences of dynamic ETMs and redundant channels have been taken into account for addressed simultaneous SUIE problem of time-varying $\mathrm{CNs}$; and 2) the newly developed estimation scheme possesses a recursive manner without dimension augmentation, hence applicable for online computations.

Remark 6: In the past decade or so, the simultaneous SUIE problem has stirred much attention and a great many results have been available in the literature. Comparing to existing results, the research carried out in this paper exhibits the following distinctive novelties: 1) the simultaneous SUIE problem is, for the first time, investigated for time-varying $\mathrm{CNs}$ subject to redundant channels and dynamic ETMs; 2) the proposed induction-based recursive approach ensures the existence of certain upper bounds on the error covariances of the unknown input and the SE; and 3) a completing-the-square approach is developed to determine the desired estimator gains so as to minimize the obtained upper bounds by solving certain recursive difference equations. 


\section{An Illustrative Example}

The CNs (1) with three nodes defined on $k \in[0,60]$ are considered with

$$
\begin{aligned}
& A_{1, k}=\left[\begin{array}{c}
0.15+0.01 \cos (2 k) \\
0.14
\end{array}\right], B_{1, k}=\left[\begin{array}{c}
0.2 \\
0.18+0.01 \cos (2 k)
\end{array}\right], \\
& A_{2, k}=\left[\begin{array}{c}
-0.2 \\
0.12+0.01 \sin (2 k)
\end{array}\right], B_{2, k}=\left[\begin{array}{c}
0.15+0.02 \cos (2 k) \\
0.1
\end{array}\right], \\
& A_{3, k}=\left[\begin{array}{c}
0.1 \\
0.1-0.01 \cos (2 k)
\end{array}\right], B_{3, k}=\left[\begin{array}{c}
0.3 \\
0.12+0.01 \sin (2 k)
\end{array}\right], \\
& \omega_{i j}=\left\{\begin{array}{ll}
-0.2, & i=j, \\
0.1, & i \neq j,
\end{array}=\operatorname{diag}\{0.1,0.1\}\right.
\end{aligned}
$$

and nonlinear function

$$
f_{k}\left(x_{i, k}\right)=E_{k} x_{i, k}+\bar{f}_{k}\left(x_{i, k}\right),
$$

where

$$
E_{k}=\left[\begin{array}{cc}
0.1 & 0.01+0.01 \cos (k) \\
0.15 & 0.2
\end{array}\right], \bar{f}_{k}\left(x_{i, k}\right)=0.01 \sin \left(x_{i, k}\right) .
$$

Then, it is easy to testify that $f_{k}(\cdot)$ satisfies (2) with $\ell_{k}=0.01$.

The unknown input $d_{i, k}(i=1,2,3)$ that needs to be estimated in (1) is given by

$$
d_{i, k}=\left\{\begin{array}{l}
1, \quad 0 \leq k \leq 30, \\
-1, \quad 31<k \leq 60 .
\end{array}\right.
$$

The number of redundant channels is $z=2$. The probabilities of the packet arrival are, respectively, set as $\bar{\pi}_{i}^{1}=0.7$ and $\bar{\pi}_{i}^{2}=0.6(i=1,2,3)$. The other parameters of the measurement output (3) are given by

$$
\begin{aligned}
& C_{1, k}^{1}=\left[\begin{array}{ll}
0.12 & 0.12+0.01 \cos (2 k)
\end{array}\right], D_{1, k}=0.15, \\
& C_{2, k}^{1}=\left[\begin{array}{ll}
0.1+0.01 \sin (2 k) & 0.18
\end{array}\right], D_{2, k}=0.3, \\
& C_{3, k}^{1}=\left[\begin{array}{ll}
0.14+0.01 \cos (2 k) & 0.16-0.01 \cos (2 k)
\end{array}\right], \\
& C_{1, k}^{2}=\left[\begin{array}{ll}
0.16 & 0.16-\sin (2 k)
\end{array}\right], D_{3, k}=0.2, \\
& C_{2, k}^{2}=\left[\begin{array}{ll}
0.18-0.01 \sin (2 k) & 0.16
\end{array}\right], F_{1, k}=0.5, \\
& C_{3, k}^{2}=\left[\begin{array}{ll}
0.17 & 0.14+0.01 \cos (2 k)
\end{array}\right], F_{2, k}=0.5 .
\end{aligned}
$$

For the dynamic event-triggered condition in (5)-(6), we choose $\gamma_{1}=\gamma_{2}=\gamma_{3}=0.2, \sigma_{1}=\sigma_{2}=\sigma_{3}=0.1, \mu_{1}=$ $\mu_{2}=\mu_{3}=10$ and $\xi_{0}^{1}=\xi_{0}^{2}=\xi_{0}^{3}=0$. The covariances of the measurement and process noises are, respectively, taken as $Q_{k}=0.1$ and $R_{k}=0.1$. The initial value $x_{i, 0}$ obeys the Gaussian distribution with the mean being zero and the covariance $P_{i, 0}^{x}=\operatorname{diag}\{0.1,0.1\}$.

With the above given parameters, according to Theorem 2 , at each time instant, the estimator parameters $K_{i, k+1}$ and $G_{i, k+1}(i=1,2,3)$ are calculated. Moreover, Figs. 1-7 present the simulation results. Figs. 1-3 depict the state trajectories and their corresponding estimates for $x_{i, k}(i=1,2,3)$, respectively. The real value of the unknown input $d_{i, k}$ and its estimate are plotted in Fig. 4. Figs. 5-6 show the trace of the minimal upper bound and the mean square error (MSE) (see [16] for its definition) for the state and the unknown input, respectively. The triggering instants of each node with the dynamic ETMs are depicted in Fig. 7. It is easily seen from Fig. 7 that the transmission frequency of measurement
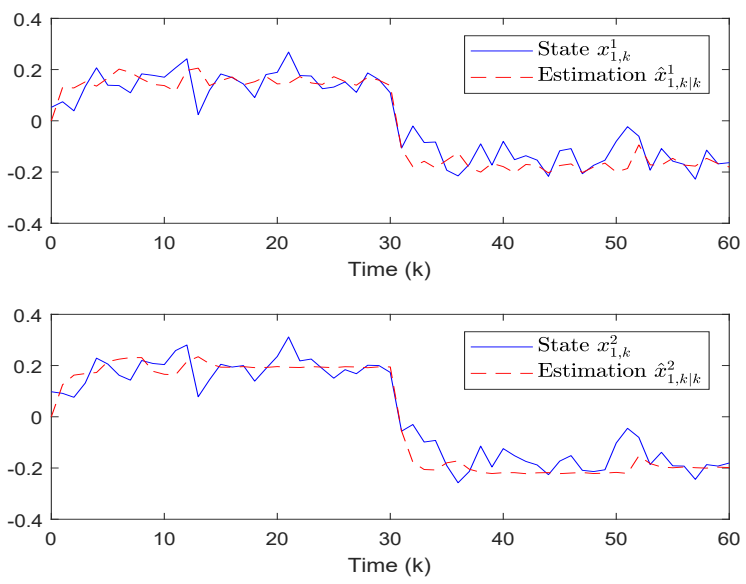

Fig. 1. State $x_{1, k}$ and its estimate $\hat{x}_{1, k \mid k}$
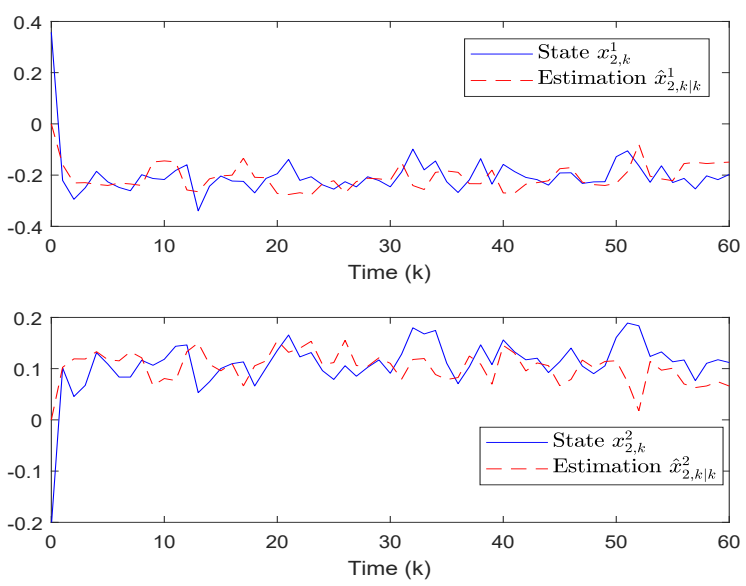

Fig. 2. State $x_{2, k}$ and its estimate $\hat{x}_{2, k \mid k}$

outputs for nodes 1, 2 and 3 is calculated as $30 \%, 41.7 \%$ and $43.3 \%$, respectively. Therefore, compared with transmitting the measurement outputs at every time instant, the dynamic ETM is capable of reducing the frequency of event releasing, thereby alleviating the energy consumption effectively. This verifies the superiority of dynamic ETMs utilized in this paper. All the simulation results have confirmed the validity of algorithm presented in this paper.

\section{CONCLUSIONS}

In this paper, the simultaneous state and input estimation issue has been discussed for discrete time-varying CNs under redundant channels and dynamic ETMs. The redundant channels have been employed to increase the transmission reliability and the dynamic event-triggered communication protocol has been introduced to save the energy cost. By using the mathematical induction, certain upper bounds on the error covariances for both the state and input estimation have been obtained and then optimized by selecting the appropriate estimator gains. The usefulness of the proposed estimation 

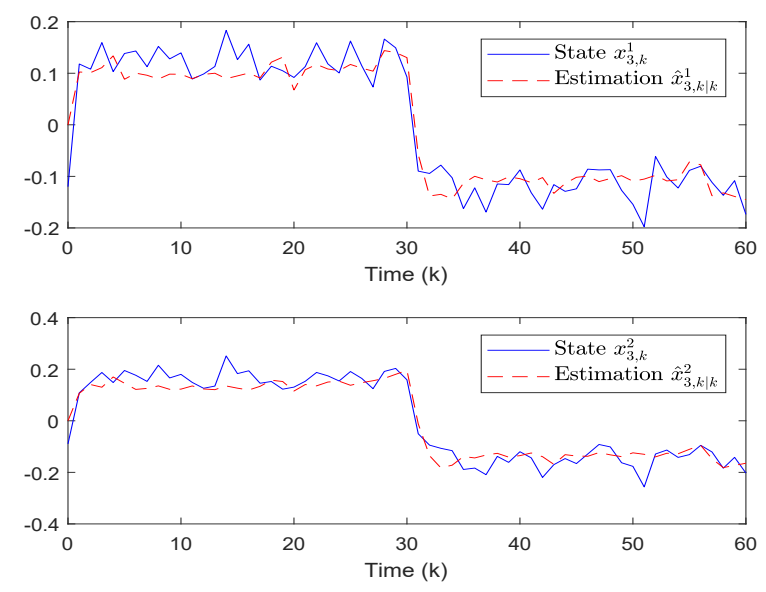

Fig. 3. State $x_{3, k}$ and its estimate $\hat{x}_{3, k \mid k}$
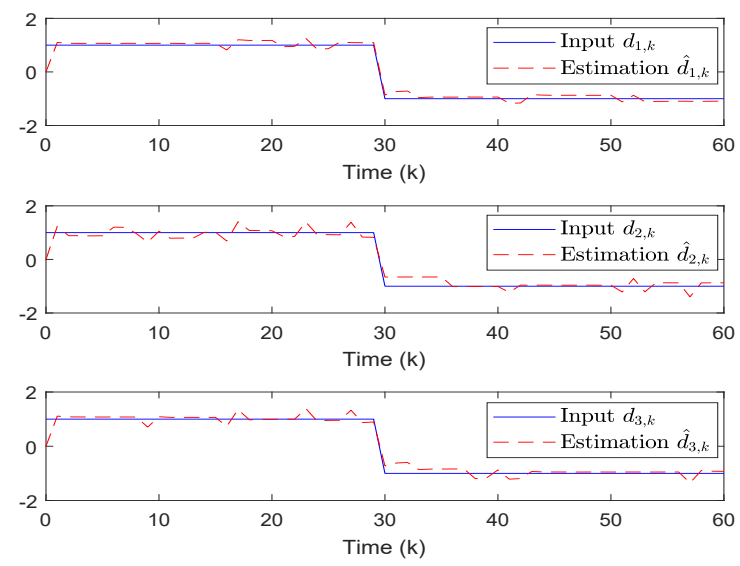

Fig. 4. Input $d_{i, k}(i=1,2,3)$ and its estimate $\hat{d}_{i, k}$
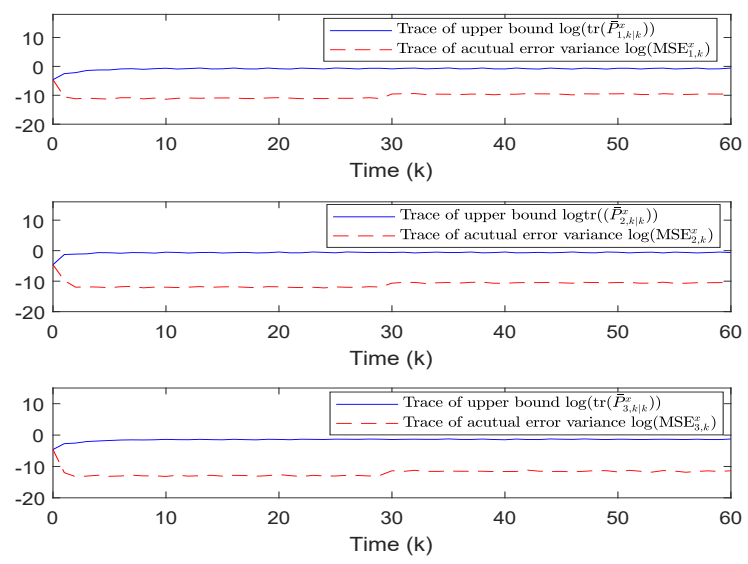

Fig. 5. Trace of SE error variance and its upper bound for nodes 1,2 and 3.
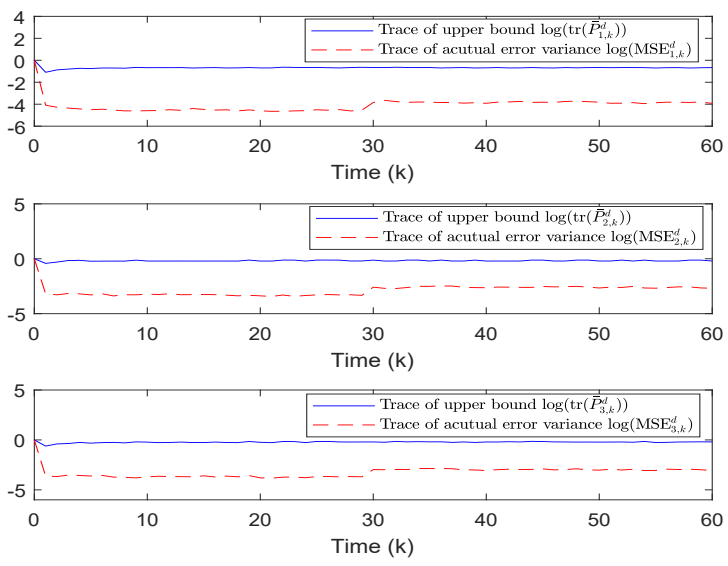

Fig. 6. Trace of input estimation error variance and its upper bound for nodes 1,2 and 3 .

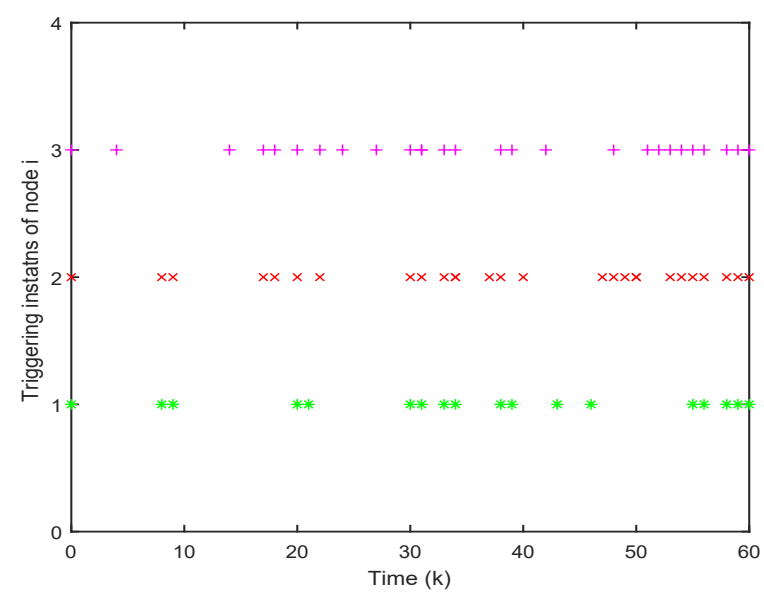

Fig. 7. The triggering instants.

scheme has been illustrated by a simulation example. Further research topics include 1) improving the estimation problem by using some novel optimization methods [26], [27] and 2) the partial-nodes-based state estimation problem of complex networks [25].

\section{REFERENCES}

[1] R. Albert, H. Jeong, and A. L. Barabasi, Error and attack tolerance of complex networks, Nature, Vol. 406, No. 6794, pp. 378-382, Jul. 2000.

[2] A. Bandyopadhyay and S. Kar, Coevolution of cooperation and network structure in social dilemmas in evolutionary dynamic complex network, Applied Mathematics and Computation, Vol. 320, pp. 710730, Mar. 2018.

[3] M. V. Basin, P. C. R. Ramirez, and F. Guerra-Avellaneda, Continuous fixed-time controller design for mechatronic systems with incomplete measurements, IEEE-ASME Transactions on Mechatronics, Vol. 23, No. 1, pp. 57-67, Feb. 2018.

[4] G. Battistelli and L. Chisci, Stability of consensus extended Kalman filter for distributed state estimation, Automatica, Vol. 68, pp. 169-178, Jun. 2016.

[5] R. Caballero-Águila, A. Hermoso-Carazo, and J. Linares-Pérez, Networked distributed fusion estimation under uncertain outputs with random transmission delays, packet losses and multi-packet processing, Signal Processing, Vol. 156, pp. 71-83, 2019. 
[6] F. S. Cattivelli and A. H. Sayed, Diffusion strategies for distributed Kalman filtering and smoothing, IEEE Transactions on Automatic Control, Vol. 55, No. 9, pp. 2069-2084, Sep. 2010.

[7] M. Chadli and H. R. Karimi, Robust observer design for unknown inputs Takagi-Sugeno models, IEEE Transactions on Fuzzy Systems, Vol. 21, No. 1, pp. 158-164, Feb. 2013.

[8] Y. Chen, Z. Wang, L. Wang and W. Sheng, Mixed $H_{2} / H_{\infty}$ state estimation for discrete-time switched complex networks with random coupling strengths through redundant channels, IEEE Transactions on Neural Networks and Learning Systems, vol 31, no. 10, pp. 4130-4142, 2020.

[9] D. Ciuonzo, A. Aubry, and V. Carotenuto, Rician MIMO channel- and jamming-aware decision fusion, IEEE Transactions on Signal Processing, Vol. 65, No. 15, pp. 3866-3880, Aug. 2017.

[10] M. Corless and J. Tu, State and Input Estimation for a Class of Uncertain Systems, Automatica, Vol. 34, No. 6, pp. 757-764, Jun. 1998.

[11] B. Demirel, V. Gupta, D. E. Quevedo, and M. Johansson, On the tradeoff between communication and control cost in event-triggered deadbeat control, IEEE Transactions on Automatic Control, Vol. 62, No. 6, pp. 2973-2980, Jun. 2017.

[12] D. V. Dimarogonas, E. Frazzol, and K. H. Johansson, Distributed event-triggered control for multi-agent systems, IEEE Transactions on Automatic Control, Vol. 57, No. 5, pp. 1291-1297, May 2012.

[13] A. Doucet, N. J. Gordon, and V. Krishnamurthy, Particle filters for state estimation of jump Markov linear systems, IEEE Transactions on Signal Processing, Vol. 49, No. 3, pp. 613-624, Mar. 2001.

[14] S. Gillijns and B. De Moor, Unbiased minimum-variance input and state estimation for linear discrete-time systems with direct feedthrough, Automatica, Vol. 43, No. 5, pp. 934-937, May 2007.

[15] A. Girard, Dynamic triggering mechanisms for event-triggered control, IEEE Transactions on Automatic Control, Vol. 60, No. 7, pp. 1992-1997, Jul. 2015.

[16] J. Hu, Z. Wang, G.-P. Liu, C. Jia and J. Williams, Event-triggered recursive state estimation for dynamical networks under randomly switching topologies and multiple missing measurements, Automatica, vol. 115, art. no. 108908, 2020.

[17] J. Hu, Z. Wang, G.-P. Liu and H. Zhang, Variance-constrained recursive state estimation for time-varying complex networks with quantized measurements and uncertain inner coupling, IEEE Transactions on Neural Networks and Learning Systems, vol. 31, no. 6, pp. 1955-1967, 2020.

[18] M. Huang and S. Dey, Stability of Kalman filtering with Markovian packet losses, Automatica, Vol. 43, No. 4, pp. 598-607, Apr. 2007.

[19] Q. Li, Z. Wang, N. Li, and W. Sheng, A dynamic event-triggered approach to recursive filtering for complex networks with switching topologies subject to random sensor failures, IEEE Transactions on Neural Networks and Learning Systems, Vol. 31, No. 10, pp. 43814388, Oct. 2020.

[20] Q. Li, B. Shen, Z. Wang, T. Huang, and J. Luo, Synchronization control for a class of discrete time-delay complex dynamical networks: a dynamic event-triggered approach, IEEE Transactions on Cybernetics, Vol. 49, No. 5, pp. 1979-1986, May 2019.

[21] H. Liu, Z. Wang, W. Fei and J. Li, Resilient $H_{\infty}$ state estimation for discrete-time stochastic delayed memristive neural Networks: A dynamic event-triggered mechanism, IEEE Transactions on Cybernetics, in press, DOI: $10.1109 /$ TCYB.2020.3021556.

[22] J. Liu, M. Yang, E. Tian, J. Cao and S. Fei, Event-based security controller design for state-dependent uncertain systems under hybridattacks and its application to electronic circuits, IEEE Transactions on Circuits and Systems I: Regular Papers, vol. 66, no. 12, pp. 4817-4828, Dec. 2019.

[23] Q. Liu, Z. Wang, X. He, and D. Zhou, Event-based recursive distributed filtering over wireless sensor networks, IEEE Transactions on Automatic Control, Vol. 60, No. 9, pp. 2470-2475, Sep. 2015.

[24] Y. Liu, Z. Wang, L. Ma and F. E. Alsaadi, A partial-nodes-based information fusion approach to state estimation for discrete-time delayed stochastic complex networks, Information Fusion, vol. 49, pp. 240-248, Sept. 2019.

[25] Y. Liu, Z. Wang, Y. Yuan and W. Liu, Event-triggered partial-nodesbased state estimation for delayed complex networks with bounded distributed delays, IEEE Transactions on Systems, Man, and Cybernetics: Systems, vol. 49, no. 6, pp. 1088-1098, Jun. 2019.

[26] Y. Liu, Q. Cheng, Y. Gan, Y. Wang, Z. Li and J. Zhao, Multi-objective optimization of energy consumption in crude oil pipeline transportation system operation based on exergy loss analysis, Neurocomputing, vol. 332, pp. 100-110, Mar. 2019.
[27] Y. Liu, S. Chen, B. Guan and P. Xu, Layout optimization of largescale oil-gas gathering system based on combined optimization strategy, Neurocomputing, vol. 332, pp. 159-183, Mar. 2019.

[28] L. Ma, Z. Wang, J. Hu and Q.-L. Han, Probability-guaranteed envelopeconstrained filtering for nonlinear systems subject to measurement outliers, IEEE Transactions on Automatic Control, in press, DOI: 10.1109/TAC.2020.3016767.

[29] A. R. Mesquita, J. P. Hespanha, and G. N. Nair, Redundant data transmission in control/estimation over lossy networks, Automatica, Vol. 48, No. 8, pp. 1612-1620, Aug. 2012.

[30] W. NaNacara and E. E. Yaz, Recursive estimator for linear and nonlinear systems with uncertain observations, Signal Processing, Vol. 62, No. 2, pp. 215-228, Oct. 1997.

[31] M. Nekovee, Y. Moreno, G. Bianconi, and M. Marsili, Theory of rumour spreading in complex social networks, Physica A-Statistical Mechanics and Its Applications, Vol. 374, No. 11, pp. 457-470, Jan. 2007.

[32] M. Nørgaard, N. K. Poulsen, and O. Ravn, New developments in state estimation for nonlinear systems, Automatica, Vol. 36, No. 11, pp. 16271638, Nov. 2000

[33] W. Qian, Y. Li, Y. Chen, and W. Liu, $L_{2}-L_{\infty}$ filtering for stochastic delayed systems with randomly occurring nonlinearities and sensor saturation, International Journal of Systems Science, vol. 51, no. 13, pp. 2360-2377, 2020.

[34] A. Selivanov and E. Fridman, Event-triggered $H_{\infty}$ control: A switching approach, IEEE Transactions on Automatic Control, Vol. 61, No. 10, pp. 3221-3226, Oct. 2016

[35] B. Shen, Z. Wang, D. Wang and Q. Li, State-saturated recursive filter design for stochastic time-varying nonlinear complex networks under deception attacks, IEEE Transactions on Neural Networks and Learning Systems, vol. 31, no. 10, pp. 3788-3800, 2020.

[36] H. Shen, S. Huo, J. Cao, and T. Huang, Generalized state estimation for Markovian coupled networks under Round-Robin protocol and redundant channels, IEEE Transactions on Cybernetics, Vol. 49, No. 4, pp. 1292-1301, Apr. 2019.

[37] B. Sinopoli, L. Schenato, M. Franceschetti, K. Poolla, M. I. Jordan, and S. S. Sastry, Kalman filtering with intermittent observations, IEEE Transactions on Automatic Control, Vol. 49, No. 9, pp. 1453-1464, Sep. 2004

[38] E. Tian, W. K. Wong, D. Yue and T.-C. Yang, $H_{\infty}$ filtering for discrete-time switched systems with known sojourn probabilities, IEEE Transactions on Automatic Control, vol. 60, no. 9, pp. 2446-2451, 2015.

[39] X. Wan, Z. Wang, M. Wu, and X. Liu, $H_{\infty}$ state estimation for discretetime nonlinear singularly perturbed complex networks under the roundrobin protocol, IEEE Transactions on Neural Networks and Learning Systems, Vol. 30, No. 2, pp. 415-426, Feb. 2019.

[40] Y. Wang, Z. Wang, L. Zou and H. Dong, $H_{\infty}$ proportional-integral state estimation for T-S fuzzy systems over randomly delayed redundant channels with partly known probabilities, IEEE Transactions on Cybernetics, in press, DOI: 10.1109/TCYB.2020.3036364.

[41] G. Wei, S. Liu, Y. Song, and Y. Liu, Probability-guaranteed setmembership filtering for systems with incomplete measurements, $A u-$ tomatica, Vol. 60, pp. 12-16, Oct. 2015.

[42] W. Xu, Z. Wang, and D. W. C. Ho, Finite-horizon $H_{\infty}$ consensus for multiagent systems with redundant channels via an observer-type eventtriggered scheme, IEEE Transactions on Cybernetics, Vol. 48, No. 5 , pp. 1567-1576, May 2018.

[43] F. Yang and Y. Li, Set-membership filtering for systems with sensor saturation, Automatica, Vol. 45, No. 8, pp. 1896-1902, Aug. 2009.

[44] J. Yang, F. Zhu, K. Yu, and X. Bu, Observer-based state estimation and unknown input reconstruction for nonlinear complex dynamical systems, Communications in Nonlinear Science and Numerical Simulation, Vol. 20, No. 3, pp. 927-939, Mar. 2015.

[45] X. Yin and D. Yue, Event-triggered tracking control for heterogeneous multi-agent systems with Markov communication delays, Journal of the Franklin Institute, vol. 350, no. 5, pp. 1312-1334, 2013.

[46] D. Zhao, Z. Wang, G. Wei and X. Liu, Nonfragile $H_{\infty}$ state estimation for recurrent neural networks with time-varying delays: On proportionalintegral observer design, IEEE Transactions on Neural Networks and Learning Systems, in press, DOI: 10.1109/TNNLS.2020.3015376.

[47] Z. Zhao, Z. Wang, L. Zou and J. Guo, Set-Membership filtering for timevarying complex networks with uniform quantisations over randomly delayed redundant channels, International Journal of Systems Science, vol. 51, no. 16, pp. 3364-3377, 2020.

[48] L. Zou, Z. Wang, H. Geng and X. Liu, Set-membership filtering subject to impulsive measurement outliers: A recursive algorithm, IEEE/CAA Journal of Automatica Sinica, vol. 8, no. 2, pp. 377-388, 2021 
[49] L. Zou, Z. Wang, J. Hu and D. H. Zhou, Moving horizon estimation with unknown inputs under dynamic quantization effects, IEEE Transactions on Automatic Control, vol. 65, no. 12, pp. 5368-5375, 2020.

[50] L. Zou, Z. Wang and D. H. Zhou, Moving horizon estimation with non-uniform sampling under component-based dynamic event-triggered transmission, Automatica, vol. 120, art. no. 109154, 2020

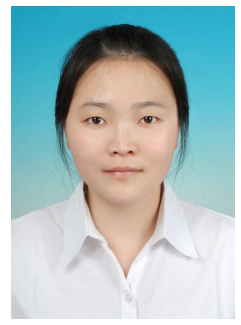

Qi Li (Member, IEEE) received her B.Eng. degree in electrical engineering and automation from Jiangsu University of Technology, Changzhou, China, in 2013 and the Ph.D. degree in control science and engineering from Donghua University, Shanghai, China, in 2018

She is currently a lecturer with the School of Information Science and Engineering, Hangzhou Normal University, Hangzhou, China. From June 2016 to July 2016, she was a Research Assistant in the Department of Mathematics, Texas A\&M University at Qatar, Qatar. From November 2016 to November 2017, she was a Visiting $\mathrm{Ph} . \mathrm{D}$. Student in the Department of Computer Science, Brunel University London, U.K. Her current research interests include network communication, complex networks and sensor networks.

Dr. Li is a very active reviewer for many international journals.

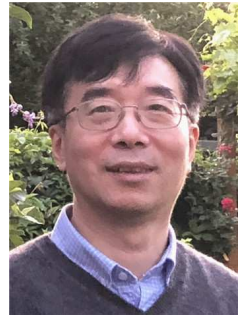

Zidong Wang (Fellow, IEEE) was born in Jiangsu, China, in 1966. He received the B.Sc. degree in mathematics from Suzhou University, Suzhou, China, in 1986, and the M.Sc. degree in applied mathematics and the Ph.D. degree in electrical engineering from the Nanjing University of Science and Technology, Nanjing, China, in 1990 and 1994, respectively.

$\mathrm{He}$ is currently a Professor of Dynamical Systems and Computing in the Department of Computer Science, Brunel University London, Uxbridge, U.K. From 1990 to 2002, he held teaching and research appointments in universities in China, Germany and the U.K. He has published more than 600 papers in international journals. He is a holder of the Alexander von Humboldt Research Fellowship of Germany, the JSPS Research Fellowship of Japan, William Mong Visiting Research Fellowship of Hong Kong. His research interests include dynamical systems, signal processing, bioinformatics, and control theory and applications.

Prof. Wang serves (or has served) as the Editor-in-Chief for the International Journal of Systems Science, the Editor-in-Chief of Neurocomputing, and an Associate Editor for 12 international journals including IEEE Transactions on Automatic Control, IEEE Transactions on Control System Technology, IEEE Transactions on Neural Networks, IEEE Transactions on Signal Processing, and IEEE Transactions on Systems, Man, and Cybernetics-Part C. He is a member of the Academia Europaea, a Fellow of the Royal Statistical Society and a member of program committee for many international conferences.

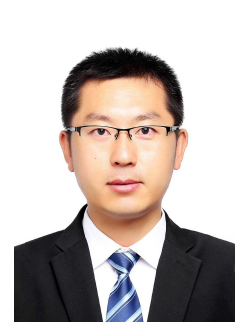

Jun Hu (Member, IEEE) received the B.Sc. degree in information and computation science and M.Sc. degree in applied mathematics from Harbin University of Science and Technology, Harbin, China, in 2006 and 2009, respectively, and the Ph.D. degree in control science and engineering from Harbin Institute of Technology, Harbin, China, in 2013.

From September 2010 to September 2012, he was a Visiting Ph.D. Student in the Department of Information Systems and Computing, Brunel University, U.K. From May 2014 to April 2016, he was an Alexander von Humboldt research fellow at the University of Kaiserslautern, Kaiserslautern, Germany. From January 2018 to January 2021, he was a research fellow at the University of South Wales, Pontypridd, U.K. He is currently a Professor in the Department of Mathematics, Harbin University of Science and Technology, China. His research interests include nonlinear control, filtering and fault estimation, time-varying systems and complex networks. He has published more than 70 papers in refereed international journals.

Dr. Hu serves as a reviewer for Mathematical Reviews, as an editor for Neurocomputing, Journal of Intelligent and Fuzzy Systems, Neural Processing Letters, Systems Science and Control Engineering, and as a guest editor for International Journal of General Systems and Information Fusion.

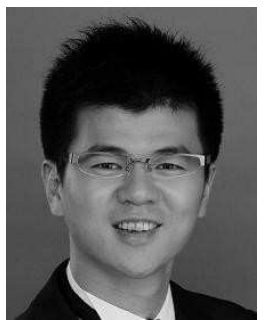

Weiguo Sheng (Member, IEEE) received the M.Sc. degree in information technology from the University of Nottingham, U.K., in 2002 and the Ph.D. degree in computer science from Brunel University, U.K., in 2005. Then, he worked as a Researcher at the University of Kent, U.K. and Royal Holloway, University of London, U.K.

He is currently a Professor at Hangzhou Normal University. His research interests include evolutionary computation, data mining/clustering, pattern recognition and machine learning. 